\title{
Statistical dispersion of flapping events in air-assisted atomization
}

\author{
Marco Crialesi-Esposito*1, Sagar Pal ${ }^{2}$, Stephane Zaleski ${ }^{2}$, Luca Brandt ${ }^{1,3}$ \\ ${ }^{1}$ FLOW Centre and SeRC, Department of Engineering Mechanics, KTH Royal Institute of \\ Technology, Stockholm, Sweden \\ ${ }^{2}$ Institut Jean le Rond d'Alembert, Sorbonne Université and CNRS, Paris, France \\ ${ }^{3}$ Department of Energy and Process Engineering, Norwegian University of Science and \\ Technology (NTNU), Trondheim, Norway \\ *Corresponding author email: marcoce@kth.se
}

\begin{abstract}
Mixing layers between air and liquid are ubiquitous in nature and extremely common in industrial applications. Among the latest, air-assisted atomization is one of the predominant forcing used to induce breakup in liquid jets. In these flows, a low-momentum liquid jet and a highmomentum air stream are injected simultaneously along the same direction, separated only by a thin solid plate. The interaction between the two phases generates an instability that manifests in the form of flapping events, during which most of the droplets are created owing to the breakup of these liquid sheets. In this study, we discuss the statistical relevance of flapping events and how they contribute to determine the final droplet-size-distribution. We present an algorithm to isolate each event in Direct Numerical Simulations and discuss the dispersion between each event. Finally, we demonstrate the importance of performing this type of analysis to reveal of statistical properties of the droplet size distribution.
\end{abstract}

\section{Keywords}

Atomization, Droplet-size-distribution, flapping events, DNS

\section{Introduction}

Air-assisted atomization is a common process used to induce fragmentation of liquid bulks. Despite its popularity in industrial applications (e.g. aeronautic combustors) many unknowns remains in the study of this process. In air-assisted atomizers, low-velocity liquid and high-velocity gas are injected along the same direction, separated only by a thin plate. At the liquid-gas interface, the velocity difference between the two phases generates a shear layer that destabilizes the liquid flow. Consequently, waves are formed in the liquid, which propagate according to the Dimotakis speed $(1,2)$. Several interfacial instabilities, such as Kelvin-Helmholtz, have been observed to contribute to the liquid breakup (2).

Air-assisted atomization has been addressed both in numerical $(2,3,4)$ and experimental studies $(5,6,7)$, while linear analysis has also been used to study the instability growth (8). Furthermore, the most unstable frequencies and corresponding wavelengths can be estimated both in experimental and numerical works (4).

Among the main unknowns in air-assisted atomizers, the most relevant in applications (e.g. combustion studies, ocean spray estimation ) is the resulting droplets-size-distribution Probability Density Function (PDF). The determination of the PDF is proven to be challenging and it has been addressed both in experiments (6) and in simulations (3). In planar air-assisted atomization, the droplets are mostly generated by wave breaking events, which are generally referred to as "flapping". These events can generate a poly-dispersed droplet distribution, and further breakup can occur as droplets interact with turbulence in the gaseous phase. Being this flow chaotic in nature, flapping events can be very different and the PDF resulting from each flapping event can be substantially altered by the local conditions.

In the present study, we investigate the statistical variations associated to flapping events by means of Direct Numerical Simulation (DNS). We start by describing the equations, the nu- 
merical method used and the simulation setup. We then discuss our main observations on the flapping events and finally the effects on the droplet size PDF.

\section{Methods}

\section{Governing equations and numerical methods}

The study has been performed using the code ParisSimulator (9). The code solves the incompressible Navier Stokes equation in the form :

$$
\partial_{i} u=0
$$

$$
\rho\left(\partial_{t} u_{i}+u_{j} \partial_{j} u_{i}\right)=-\partial_{i} p+\partial_{i}\left[\mu\left(\partial_{i} u_{j}+\partial_{j} u_{i}\right)\right]+\sigma \kappa \delta_{s} n_{i}
$$

where $u_{i}$ is the velocity vector, $p$ the pressure, $\rho$ the density and $\mu$ the viscosity. The last term on the right-hand-side of Equation (1b) is the surface tension term, where $\sigma$ si the surface tension coefficient, $\kappa$ is the curvature, $n_{i}$ is the surface normal and $\delta_{s}$ is a Dirac-delta function used to concentrate the force at the interface. The different phases are captured through the Volume of Fluid (VOF) method, where an advection equation for a color function $C$ is used:

$\partial_{t} C+u \cdot \partial_{i} C=0$.

The color function is $C=1$ in the liquid phase and $C=0$ in the gas phase and allows to compute the local density an viscosity by arithmetic mean as:

$$
\begin{aligned}
& \rho=C \rho_{l}+(1-C) \rho_{g} \\
& \mu=C \mu_{l}+(1-C) \mu_{g}
\end{aligned}
$$

where the subscripts $l$ and $g$ represents the liquid and gas phase.

The equations are solved on a Cartesian staggered grid (with fixed cuboid cells), with the projection-method used to obtain a variable-coefficient Poisson equation for $p$, solved using the multigrid solver HYPRE. The temporal integration is performed using a second-order predictorcorrector method on a mass-momentum consistent formulation (10). The diffusion term is computed explicitly with a second-order centered scheme. Finally, the surface normal is computed using the Mixed-Young-Centered-Scheme and the curvature using the Height-Function method from (11).

\section{Case setup}

The simulation performed in this study is the M2-A20 case discussed in $(2,3)$. i.e. a parallel planar two-phase mixing-layer. The fluids properties for the case studied are presented in Table 1, while the value of the geometrical parameters can be found in Table 2. Liquid and gas are injected with velocities $U_{l}$ and $U_{g}$ (along the $\hat{x}$ direction), through confining gaps of height $H_{l}$ and $H_{g}$, disposed in the vertical direction $(\hat{y})$. The two phases are separated by a thin solid plate of thickness $l_{y}$ and the setup is chosen so that $H_{l}=H_{g}+l_{y}$. Downstream of the inlet, two vorticity layers are formed, with quiescent gas $\delta_{g}$ and with the liquid $\delta_{l}$ and they are assumed to be equal in this study (2). The domain streamwise $\left(L_{x}\right)$, normal $\left(L_{y}\right)$ and spanwise $\left(L_{y}\right)$ dimensions are chosen consistently with the reference study (2). As described in (3), M2 corresponds to the case where $H_{l} / \Delta x=128$, where $\Delta x$ is the grid size, leading to $2048 \times 1024 \times 256$ grid points in each direction.

The dynamic pressure ratio between the two phases is $M=\rho_{g} U_{g}^{2} /\left(\rho_{l} U_{l}^{2}\right)=20$. It has been proved in several studies $(4,12)$ that this parameter is dictating the large-scale dynamics and stability of the two phase mixing layer. A large value of $M$ is required to ensure the absolute instability regime, which is indeed reached for the present setup $(2,13)$. The gas-phase Reynolds number is $R e_{g}=\rho_{g} U_{g} H_{g} / \mu_{g}=7750$ which ensures the fully turbulent behavior of the gaseous stream. 
Table 1. Physical properties of liquid and gas.

\begin{tabular}{ccccccc}
$\rho_{l}$ & $\rho_{g}$ & $\mu_{l}$ & $\mu_{g}$ & $\sigma$ & $U_{l}$ & $U_{g}$ \\
\hline 1000 & 50 & $10^{-3}$ & $5 \cdot 10^{-5}$ & 0.05 & 0.5 & 20 \\
\hline$\left[\mathrm{kg} / \mathrm{m}^{3}\right]$ & {$\left[\mathrm{kg} / \mathrm{m}^{3}\right]$} & {$[P a s]$} & {$[P a s]$} & {$[\mathrm{N} / \mathrm{m}]$} & {$[\mathrm{m} / \mathrm{s}]$} & {$[\mathrm{m} / \mathrm{s}]$}
\end{tabular}

Table 2. Simulation geometrical setup.

\begin{tabular}{cccccc}
$H_{l}$ & $l_{y}$ & $L_{x} / H_{l}$ & $L_{y} / H_{l}$ & $L_{z} / H_{l}$ & $\delta_{g}, \delta_{l}$ \\
\hline $8 \cdot 10^{-4}$ & $2.5 \cdot 10^{-5}$ & 16 & 8 & 2 & $1 \cdot 10^{-4}$ \\
\hline$[\mathrm{m}]$ & {$[\mathrm{m}]$} & - & - & - & {$[\mathrm{m}]$}
\end{tabular}

\section{Results and discussion}

\section{Flapping events and temporal statistics.}

The main focus of this work is on droplet generation and statistics, which are tightly related to flapping events. During flapping, a wave-like liquid structure breaks into several droplets. This event can be qualitatively observed in Figure 1, where we display the liquid-gas interface by the value 0.5 of the volume of fluid indicator function. The vorticity field is displayed in the background wallnormal-streamwise plane together with the inflow velocity profile in the inlet cross-stream plane. The flow is indeed forming both large and small scale turbulent structures, where a clear scale separation can be observed (quantified in previous studies (2)).

In this flapping event, we can clearly observe that a significant amount of liquid is teared by the gas and breaks into droplets, which are rapidly advected towards the outflow boundary by the gas stream. This is a fundamental aspect as, both in numerical simulations and experiments, the droplet detection (on which statistics are based) is heavily influenced by the choice of the region where the analysis is performed. In experiments, the need for appropriate optical focus (able to resolve small droplets contours) forces to reduce the visualization field to a specific region of the domain, while in numerical simulations, a similar approach is used to limit the amount of data analyzed (3). Both the main temporal frequencies and wavelengths should be estimated. The interfacial wave wavelength $\lambda$ can be measured directly from simulation snapshots (2) and for this case $\lambda=4.5 H_{l}$. The most unstable frequency in the problem can be found as $f_{0}=U_{D} / \lambda$ where $U_{D}$ is the Dimotakis velocity, computed as:

$$
U_{D}=\frac{U_{g} \sqrt{\rho_{g}}+U_{l} \sqrt{\rho_{l}}}{\sqrt{\rho_{l}}+\sqrt{\rho_{g}}} .
$$

If we consider a timescale $H_{l} / U_{g}$, the resulting frequency $f_{0} H_{l} / U_{g} \approx 0.05$ (2). Even from a qualitative look at Figure 1, it is evident that flapping events spread droplet over the whole domain and that fragmentation is still occurring far from the liquid bulk interface. It is therefore clear that the vision field needs to be significantly larger than $\lambda$, to capture both breakup and coalescence far from where the droplets are generated.

The characteristic frequencies can be studied by analyzing the number of droplets $N_{d}$ over time, showed in Figure 2 against the dimensionless time $t U_{g} / H_{l}$. Here, all the droplets in the domain are detected, regardless of their position, shape, size or speed. A clear periodicity can be observed, which can be related with the flapping events. As example, we isolated a single flapping event in the top panel. At first, the wave is formed (red box). As the wave flapping occurs, droplets start being generated and their number eventually reaches a peak once the wave fully breaks (blue box). Droplets are then transported outside the domain by the gas stream and the droplets count consistently decreases. The whole process takes approximately $t \approx 2 / f_{0}$, but a lag between each consecutive flapping events can be observed, which lasts $t \approx 1 / f_{0}$. This lag suggests that the frequency $f_{0}$, resulting from the Dimotakis speed, may not represents faithfully the frequency of these flapping events. 


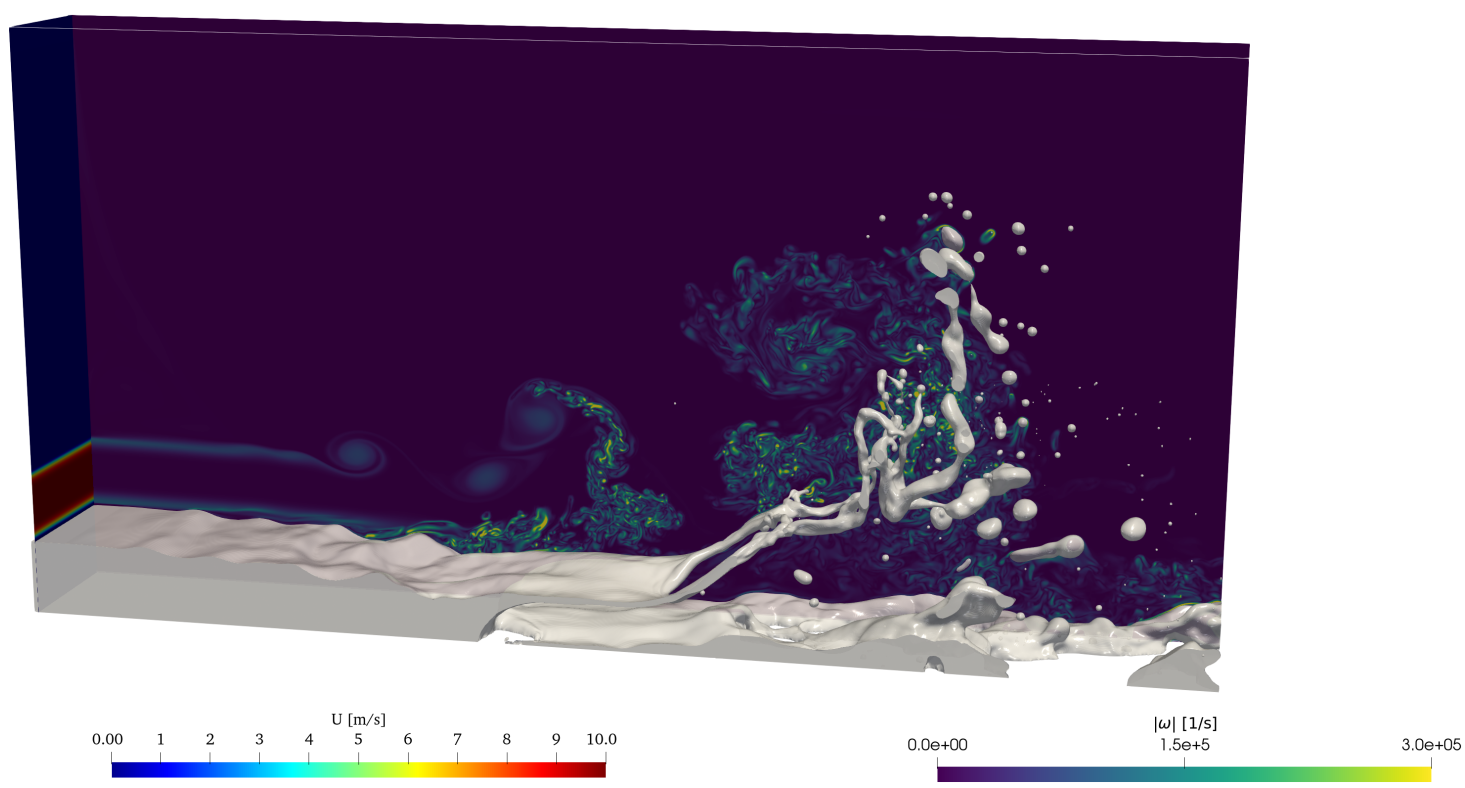

Figure 1. A flapping event: In white a render of the interface defined by the value 0.5 of the indicator function. The vorticity field is displayed in the background wallnormal-streamwise plane together with the inflow velocity profile in the inlet cross-stream plane.

While some events can be clearly identified, others are more chaotic, which is reasonable given the turbulent nature of the flow. For this reason, in the following sections, we consider as flapping an event occurring between two local minima of the droplet temporal distribution in Figure 2, while plateau close to the minimum values are not considered in the analysis.

\section{Droplet size distributions}

We discuss now the droplet size distribution PDF, with a particular focus on how the flapping events influence the standard deviation for each droplet size interval. The PDF of each flapping event is computed as:

$$
P D F_{i}(d /\langle d\rangle)=\frac{N_{j, i}}{N_{t o t, i} \delta_{b, j}}
$$

where the subscripts $i$ and $j$ indicate the $i$ th flapping event and the $j$ th bin, $N_{j, i}$ is the number of droplets generated in the bin $j$ by the event $i, N_{t o t, i}$ is the total number of droplets generated in the flapping event $i$ and $\delta_{b, j}$ is the width of the $j$ bin. By maintaining a constant binning for all events, we can compute the standard deviation $\sigma_{j}$ for the $j$ bin.

We consider the three statistical distributions mostly addressed in literature, namely the lognormal $P_{l o g n}$, the gamma distribution $P_{\Gamma}$ and the n-gamma $P_{n \Gamma}$, defined as:

$$
P_{\operatorname{logn}}(x)=\frac{1}{x \hat{\sigma} \sqrt{2 \pi}} \exp \left[\frac{-(\ln x-\hat{\mu})^{2}}{2 \hat{\sigma}^{2}}\right] ; \quad P_{\Gamma}(x)=\frac{\beta^{\alpha} x^{\alpha-1}}{\Gamma(\alpha)} \exp (-\beta x)
$$

where $x=d /\langle d\rangle, \hat{\mu}$ and $\hat{\sigma}$ are the mean and standard deviation of $\ln x, \alpha=(\mu / \sigma)^{2}$ (with $\mu$ and $\sigma$ are mean and standard deviation of to $x$ ) and $\beta=\alpha / \mu . P_{n \Gamma}$ can be obtained from $P_{\Gamma}$ with $n=\alpha$ and is valid only when the distribution is computed over a variable normalized by its mean, so that the parameter $\beta$ disappears. $P_{n \Gamma}$ is usually fitted onto distributions obtained experimentally and $n$ is used a fitting parameter (6).

Figure 3(a) shows the PDF obtained with the present simulation. In black, we display the PDF computed for all droplets generated during all flapping events, blue error-bars indicate to the standard deviation of each droplet size, and in green the line marking $d=8 \Delta X$. Dashed 


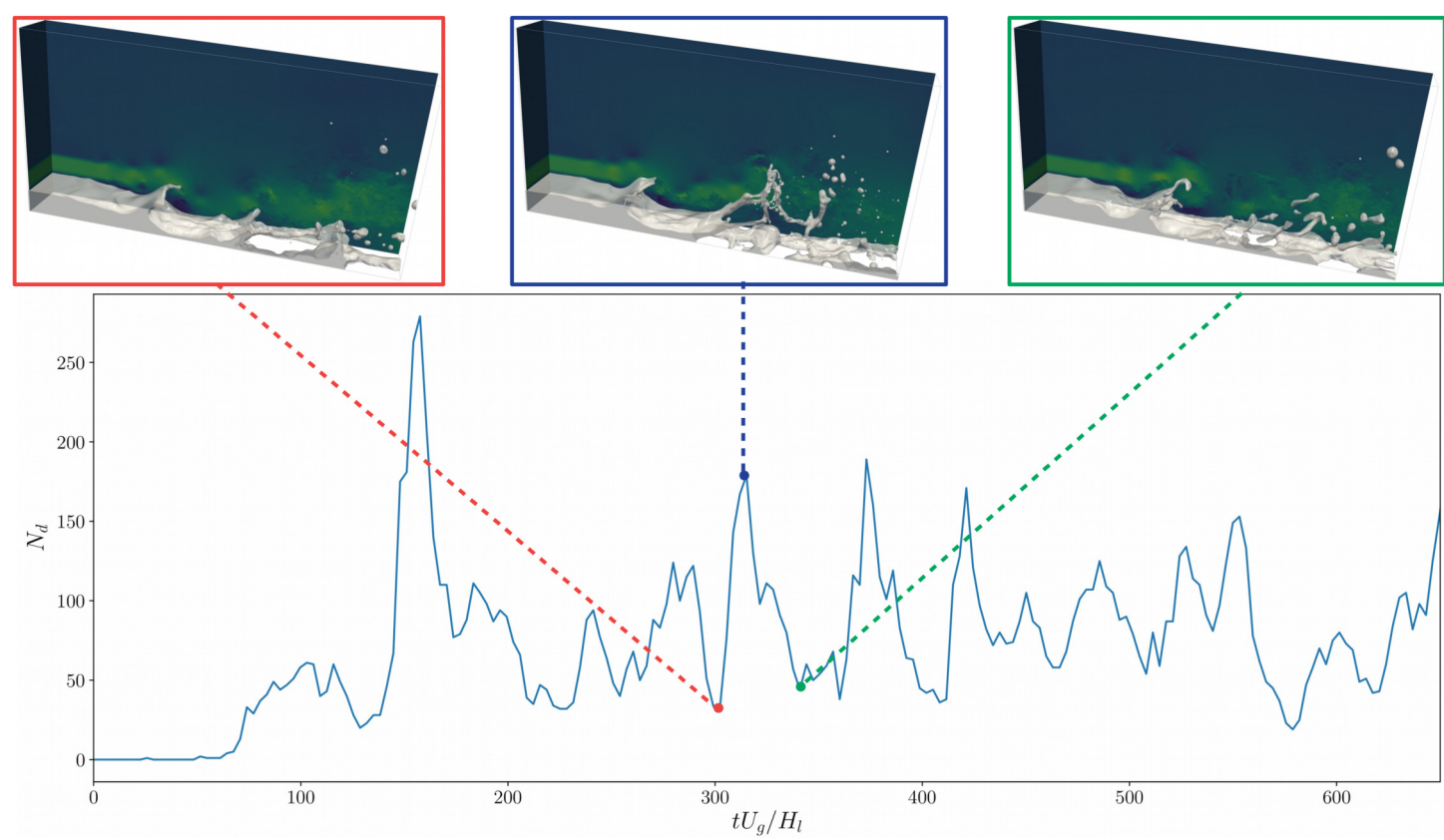

Figure 2. Number of droplet $N_{d}$ generated over time, normalized using the gas velocity $U_{g}$ and the liquid gap height $H_{l}$. The top panels display snapshot of the temporal sequence of a flapping event.

lines represent the statistical distributions discussed above, where $P_{l o g n}$ and $P_{\Gamma}$ are directly obtained from the droplets datasets (i.e. by computing mean and standard deviation), while $P_{\Gamma}$ is a fit to the data. All droplets with $d \leq 4 \Delta x$ have been removed from the analysis, as these are generally considered debris (14). Most of the droplets detected are well resolved, with a mean diameter $\langle d\rangle \approx 0.24 H_{l}$; a significant scale separation is observed in the distribution. We measured $\hat{\sigma} \approx 1.25$, which is approximately $5 \%$ larger than what observed in previous studies for the same configuration (3). The reason for this discrepancy (although small) lies probably in the portion of the domain in which droplets are counted. As in the present study the whole domain is analyzed (unlike in (3)), we are able to capture any breakup occurring during flapping events, hence enlarging the amount of droplets detected and increasing scale separation.

The variation between flapping events is small for $d<\langle d\rangle$, suggesting that the breakup process is similar for all flapping events. The small standard deviation at these scales suggests that in any flapping event, at small scales breakup develops in a deterministic way, likely due to the developed turbulence field. For larger scales $(d>\langle d\rangle)$, error-bars increase significantly and the process becomes more chaotic. While this is partially due to a more limited statical sampling (i.e. based on less frequent events) it is also likely that this is the results of the chaotic growth of concurrent large scale instabilities. Hence, events like coalescence and formation of large droplets appear more randomly than breakup at small scales and their period might be significantly longer than expected and difficult to estimate.

In addition, we observe a clear overlapping of the simulation data with the log-normal distribution, which is consistent with previous studies $(3,15)$. Gamma distributions seem to reproduce the distribution less accurately, although $\mathrm{n}-$ Gamma captures well the PDF around $d=\langle d\rangle$. Furthermore, the n-Gamma is remarkably close to the error-bar ranges for larger droplets $(d\rangle\langle d\rangle)$, suggesting that further investigation on large scale events is required to understand how distributions describe these events.

Finally,in Figure 3(b), we show the mass-based PDF defined as:

$$
\operatorname{PDF}\left(M_{d}\right)=\frac{m_{j}}{M_{t o t} \delta x}
$$

where $m_{i}$ is the mass associated with the $i$ droplet and $M_{t o t}$ is the total droplet mass (all droplets measured over time). The same approach described before can be used to compute 

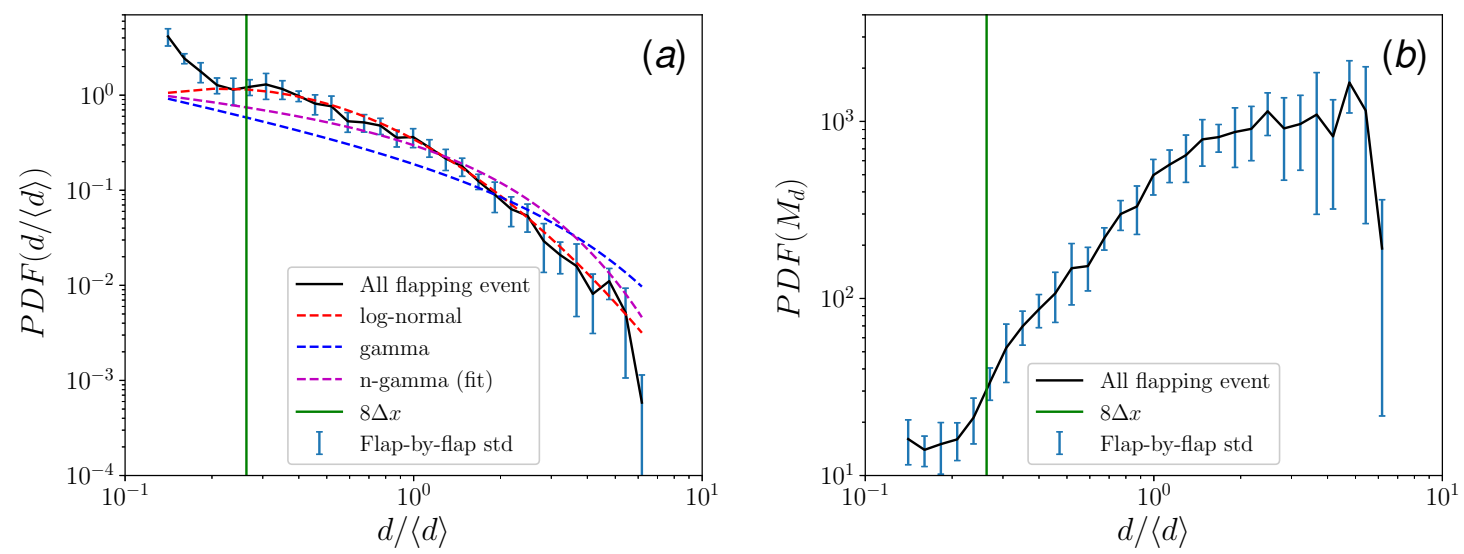

Figure 3. Droplet size distribution. (a) PDF of the droplet size. The continuous black line shows the PDF for all the flapping event considered, blue error-bars represents the standard deviation among all flapping events. Dashed lines represent statistical distributions proposed to describe the process, namely log-normal (red), gamma (blue) and n-gamma (magenta). Finally, the vertical green line represent the point where $d=8 \Delta x$. (b) mass-based PDF of the droplet distribution with same line style as for panel (a).

$\sigma_{j}$ for to the mass. As expected, the standard deviation is amplified in the flapping events and becomes increasingly more relevant for larger droplets. Again, for $d\rangle\langle d\rangle$ the standard deviation progressively increases, reaching almost one order of magnitude, highlighting how important is to focus on this region of the PDF in future studies.

A final remark can be made on the accuracy of the distributions. It is well known that the lognormal one is generally associated with fragmentation processes, while the Gamma distribution is derived from coalescence processes (16). For $d<\langle d\rangle$ the standard deviation observed between flapping events seems to be small for both mass and diameter, suggesting that breakup events are dictated by consistent dynamics, easily captured by the temporal statistics. On the other hand, large droplets are rare events, the product of coalescence and larger scale instabilities. Hence, small variations in the right-tail of the distribution could lead to significant differences in the mean and standard deviation, leading to a better agreement between the PDF and either Gamma or log-normal statistical models which is biased by the limited statistics. Large droplets are difficult to capture both with numerical simulations and in experimental works, as they require to enlarge the portion of the domain analyzed and the way in which droplets are detected. It is therefore our opinion that future studies should focus on accurately describing the large droplet generation, potentially requiring to capture a large number of flapping events where these are formed.

\section{Conclusions}

We performed a DNS study of an air-assisted atomizer aiming to analyze the relevance of flapping on the droplet size distribution. The simulation was performed in conditions relevant to realistic applications (i.e. high dynamic pressure ratio and high Reynolds number). We have analyzed the droplet generation over time and shown how it is possible to isolate flapping events. We also examined the droplets generated during each flapping event and computed the standard deviation on each bin of the PDF of the total droplet size.

Our observations suggest that flapping events occur on a time interval $t=2 / f_{0}$, but the frequency resulting from the Dimotakis speed and the wavelength do not seem to be a good descriptor of the flapping frequencies. This suggests that in order to understand droplets temporal dynamics, further studies will have to account for a more accurate time-analysis of wave stretching and breakup.

In the analysis of the PDF, we found very small dispersion between different flapping events for small droplets, which reveals both the accurate description of the breakup process and a large enough droplet population leading to accurate statistics. On the other hand, large droplets $(d>\langle d\rangle)$ suffer from significant statistical dispersion. This shows that their formation 
is a rare process and that many flapping events are needed in order to reach low statistical dispersion. Furthermore, several factors concur in the formation of large droplets. In a first place, large-scale turbulent motions (i.e. large vortices) are tightly related to wave formation and breakup. Also, coalescence of small droplets, although less likely to occur in flapping events, may generate large droplets. Finally surface instabilities are formed, which lead to complex phenomena. The non-linear combination of all these factors and their characteristic timescales lead to significant statistical dispersion for large droplets.

We believe that, in future studies, focus should be put on the analysis of large liquid structures and in defining accurate techniques and experimental setups that may help to gain additional understanding on the larger-size droplets. As these contain a significant part of the liquid mass, its accurate description is fundamental in applications such as combustion and in describing environmental processes.

\section{Acknowledgements}

M.C.E. and L.B. acknowledge the support from the Swedish Research Council via the multidisciplinary research environment INTERFACE, Hybrid multiscale modelling of transport phenomena for energy efficient processes and the Grant No. 2016-06119. The computer time was provided by the National Infrastructure for High Performance Computing and Data Storage in Norway (project no. NN9561K). SZ and SP acknowledge the support from HPC resources of CINES under the allocations 2018-A0052B07760 and 2019-A0072B07760 and the resources of the TGCC under the project 2020225418 granted respectively by GENCI and PRACE and by the Flash Covu. Support by the ERC ADV grant TRUFLOW and by the Fondation de France for the ANR action Flash Covid is also acknowledged.

\section{References}

[1] P. E. Dimotakis, "Entrainment and growth of a fully developed, two-dimensional shear layer," AIAA J, vol. 24, pp. 1791-1796, 1986.

[2] Y. Ling, D. Fuster, G. Tryggvason, and S. Zaleski, "A two-phase mixing layer between parallel gas and liquid streams: Multiphase turbulence statistics and influence of interfacial instability," Journal of Fluid Mechanics, vol. 859, pp. 268-307, jan 2019.

[3] Y. Ling, D. Fuster, S. Zaleski, and G. Tryggvason, "Spray formation in a quasiplanar gasliquid mixing layer at moderate density ratios: A numerical closeup," Physical Review Fluids, vol. 2, no. 1, p. 014005, 2017.

[4] D. Fuster, J. P. Matas, S. Marty, S. Popinet, J. Hoepffner, A. Cartellier, and S. Zaleski, "Instability regimes in the primary breakup region of planar coflowing sheets," Journal of Fluid Mechanics, vol. 736, pp. 150-176, 2013.

[5] J.-p. Matas, S. Marty, M. S. Dem, and A. Cartellier, "Influence of Gas Turbulence on the Instability of an Air-Water Mixing Layer," vol. 074501, no. August, pp. 1-5, 2015.

[6] P. H. Marmottant and E. Villermaux, "On spray formation," Journal of Fluid Mechanics, vol. 498, pp. 73-111, jan 2004.

[7] J.-p. Matas, A. Delon, and A. Cartellier, "Shear instability of an axisymmetric air - water coaxial jet," pp. 575-600, 2018.

[8] T. Boeck, P. Yecko, A. Bague, and S. Zaleski, "Instability of Two-Phase Mixing Layers," in Proceeding of ICMF07, (Leipzig), 2007.

[9] W. Aniszewski, T. Arrufat, M. Crialesi-Esposito, S. Dabiri, D. Fuster, Y. Ling, J. Lu, L. Malan, S. Pal, R. Scardovelli, et al., "Parallel, robust, interface simulator (paris)," Computer Physics Communications, vol. 263, p. 107849, 2021. 
[10] T. Arrufat, M. Crialesi-Esposito, D. Fuster, Y. Ling, L. Malan, S. Pal, R. Scardovelli, G. Tryggvason, and S. Zaleski, "A mass-momentum consistent, volume-of-fluid method for incompressible flow on staggered grids," Computers \& Fluids, vol. 215, p. 104785, 2021.

[11] S. Popinet, "An accurate adaptive solver for surface-tension-driven interfacial flows," Journal of Computational Physics, vol. 228, no. 16, pp. 5838-5866, 2009.

[12] J. C. Lasheras and E. Hopfinger, "Liquid jet instability and atomization in a coaxial gas stream," Annual review of fluid mechanics, vol. 32, no. 1, pp. 275-308, 2000.

[13] J.-P. P. Matas, S. Marty, and A. Cartellier, "Experimental and analytical study of the shear instability of a gas-liquid mixing layer," Physics of Fluids, vol. 23, no. 9, p. 94112, 2011.

[14] J. Shinjo and A. Umemura, "Simulation of liquid jet primary breakup: Dynamics of ligament and droplet formation," International Journal of Multiphase Flow, vol. 36, pp. 513-532, jul 2010.

[15] C. Pairetti, R. Villiers, and S. Zaleski, "A numerical cough machine," arXiv preprint arXiv:2101.05662, 2021.

[16] E. Villermaux, "Fragmentation," Annual Review of Fluid Mechanics, vol. 39, pp. 419-446, jan 2007. 